\title{
Gauge/String Duality and Hadronic Physics
}

\author{
Henrique Boschi-Filho and Nelson R. F. Braga \\ Instituto de Física, Universidade Federal do Rio de Janeiro, Caixa Postal 68528, RJ, 21941-972, Brazil
}

Received on 24 March, 2006

We review some recent results on phenomenological approaches to strong interactions inspired in gauge/string duality. In particular, we discuss how such models lead to very good estimates for hadronic masses.

Keywords: AdS/CFT; String theory; QCD

\section{INTRODUCTION}

Let us start by briefly reviewing some early results on strong interactions which lead to the proposal of string theory. These results date back to the decade of 1960. The first is the experimental observation, in hadronic scattering, of an apparently infinite tower of resonances with mass and angular momenta related by

$$
J \sim m^{2} \alpha^{\prime}
$$

where $\alpha^{\prime} \sim 1(\mathrm{GeV})^{-2}$ is the Regge slope.

Another important fact is that the properties of hadronic scattering in the so called Regge regime are nicely described by the amplitude postulated by Veneziano (in terms of Mandelstan variables $(s, t, u))$

$$
A(s, t)=\frac{\Gamma(-\alpha(s)) \Gamma(-\alpha(t))}{\Gamma(-\alpha(s)-\alpha(t))}
$$

where $\Gamma(z)$ is the Euler gamma function and $\alpha(s)=\alpha(0)+$ $\alpha^{\prime} s$.

A strong motivation for relating hadrons to strings is the fact that these two results of eqs. (1) and (2) can be reproduced from a relativistic bosonic string[1, 2]. A relativistic bosonic string can be described by the action

$$
S=-\frac{1}{4 \pi \alpha^{\prime}} \int d \tau d \sigma \sqrt{-g} g^{a b} \partial_{a} X^{\mu} \partial_{b} X_{\mu}
$$

After quantization, one finds that the spectrum of excitations shows up in representations satisfying eq. (1). The string scattering amplitudes also reproduce the Veneziano result eq. (2).

On the other hand, some problems concerning a possible string description of hadronic physics have been a challenge for physicists for a long time. One of them is the behavior of the scattering amplitudes at high energy. If one considers the Regge limit, corresponding to $s \rightarrow \infty$, with fixed $t:$ the Veneziano amplitude behaves as

$$
A \sim s^{\alpha(t)}
$$

where $\alpha(t)=\alpha(0)+\alpha^{\prime} t$, in agreement with experimental results. Actually this was one of the inputs for building up the
Veneziano amplitude. However considering high energy scattering at fixed angles that correspond to the limit $s \rightarrow \infty$ with $s / t$ fixed the amplitude behaves as (soft scattering)

$$
A_{\text {Ven. }} \sim \exp \left\{-\alpha^{\prime} s f(\theta)\right\}
$$

while experimental results for hadrons show a hard scattering behavior

$$
A_{\text {exp. }} \sim s^{(4-\Delta) / 2},
$$

that is reproduced by QCD $[3,4]$.

As we will discuss later, this apparent obstacle to describing strong interactions using string theory was solved recently by Polchinski and Strassler[5].

In the mean time it was realized that string theory contains massless spin two excitations that can be associated with the graviton. This allows one to interpret string theory as a quantum theory for gravity. In fact the symmetry groups for the possible string theories are large enough to contain also all the fields of the standard model. So presently string theory is a candidate for a unified theory for all interactions.

In this review we are going to discuss recent results concerning the relation between string theory and QCD. QCD has been tested and confirmed with success in high energy experiments. But QCD is non perturbative at low energies. Lattice calculations give us very important results in this regime. However it seems that we are still far from a complete description of the complexity of strong interactions. In particular, important aspects like confinement and mass generation still lack of a satisfactory description. Presently there are many indications that string theory can be useful in the description of strong interactions in the non perturbative regimes of QCD.

An early connection between $\mathrm{SU}(\mathrm{N})$ gauge theories (for large $\mathrm{N}$ ) and string theory was realized long ago by 't Hooft[6]. A few years ago a very important result was obtained by Maldacena[7] relating string theory in anti-de Sitter (AdS) space with gauge theories in Minkowski space. He established a correspondence between string theory in $A d S_{5} \times S^{5}$ space-time and $\mathcal{N}=4$ superconformal Yang Mills $\mathrm{SU}(\mathrm{N})$ theory for large $\mathrm{N}$ in its four dimensional boundary. This is known as AdS/CFT correspondence[7-9].

In the AdS/CFT correspondence we have an exact duality between a four dimensional gauge theory and string theory in a ten dimensional space. However, in this formulation, the gauge theory has no energy scale as it is conformal. There are 
many attempts to search for exact dualities involving gauge theories more similar to QCD[10-13].

Although it involves a conformal gauge theory, the AdS/CFT correspondence has been a very important source of inspiration for searching QCD results from string theory. The first idea of breaking conformal invariance in the AdS/CFT context, proposed by Witten, is to consider an AdS Schwarzschild black hole as dual to a non-supersymmetric Yang Mills theory[14]. This approach was used to calculate glueball masses in [15-21].

\section{QCD SCATTERING AND STRING THEORY}

Recently Polchinski and Strassler[5] introduced an infrared cut off in the AdS space and reproduced the hard scattering behavior of strong interactions at fixed angles from string theory. Inspired in the AdS/CFT correspondence they assumed a duality between gauge theory glueballs and string theory dilatons in an AdS slice and found the QCD scaling.

This scaling was also obtained in [22] from a mapping between quantum states in AdS space and its boundary found in [23]. We considered an AdS slice as approximately dual to a confining gauge theory. The slice corresponds to the metric

$$
d s^{2}=\frac{R^{2}}{(z)^{2}}\left(d z^{2}+(d \vec{x})^{2}-d t^{2}\right)
$$

with $0 \leq z \leq z_{\max } \sim 1 / \mu$ where $\mu$ is an energy scale chosen as the mass of the lightest glueball. We used a mapping between Fock spaces of a scalar field in AdS space and operators on the four dimensional boundary, defined in [23]. Considering a scattering of two particles in $m$ particles one finds a relation between bulk and boundary scattering amplitudes[22]

$$
S_{\text {Bulk }} \sim S_{\text {Bound. }}\left(\frac{\sqrt{\alpha^{\prime}}}{\mu}\right)^{m+2} K^{(m+2)(1+d)}
$$

where $d$ is the scaling dimension of the boundary operators and $K$ is the boundary momentum scale. This leads to the result for the amplitude

$$
A_{\text {Boundary }} \sim s^{(4-\Delta) / 2},
$$

where $\Delta$ is the total scaling dimension of scattered particles. This reproduces the QCD scaling of eq. (6).

For some other results concerning QCD scattering properties from string theory see also [24-30].

\section{SCALAR GLUEBALL MASSES}

Using the phenomenological approach of introducing an energy scale by considering an AdS slice we found estimates for scalar glueball mass ratios[31, 32]. In the $\mathrm{AdS}_{5}$ bulk we took dilaton fields satisfying Dirichlet boundary conditions at $z=z_{\max }$

$$
\begin{aligned}
\Phi(z, \vec{x}, t) & =\sum_{p=1}^{\infty} \int \frac{d^{3} k}{(2 \pi)^{3}} \frac{z^{2} J_{2}\left(u_{p} z\right)}{z_{\max } w_{p}(\vec{k}) J_{3}\left(u_{p} z_{\max }\right)} \\
& \times\left\{\mathbf{a}_{p}(\vec{k}) e^{-i w_{p}(\vec{k}) t+i \vec{k} \cdot \vec{x}}+\text { h.c. }\right\},
\end{aligned}
$$

where $w_{p}(\vec{k})=\sqrt{u_{p}^{2}+\vec{k}^{2}}$ and the functions $J_{v}$ with $v=2,3$ are Bessel functions of order $v$. The momentum associated with the $z$ direction is

$$
u_{p}=\frac{\chi_{2, p}}{z_{\max }}
$$

where $\chi_{2, p}$ are the zeroes of the Bessel functions: $J_{2}\left(\chi_{2, p}\right)=0$.

On the boundary $(z=0)$ we considered scalar glueball states $J^{P C}=0^{++}$and their excitations $0^{++*}, 0^{++* *}$ with masses $\mu_{p}, p=1,2, \ldots$ Assuming an approximate gauge/string duality the glueball masses are taken as proportional to the dilaton discrete modes:

$$
\frac{u_{p}}{\mu_{p}}=\text { const. }
$$

So, the ratios of glueball masses are related to zeros of the Bessel functions

$$
\frac{\mu_{p}}{\mu_{1}}=\frac{\chi_{2, p}}{\chi_{2,1}}
$$

Note that these ratios are independent of the size of the slice $z_{\max }$. Our estimates compared with $S U(3)$ lattice $[33,34]$ and AdS-Schwarzshild [15] (in GeV) are shown in Table I.

\begin{tabular}{|l|c|c|c|}
\hline $4 \mathrm{~d}$ State & lattice, $N=3$ & AdS-BH & AdS slice \\
\hline $0^{++}$ & $1.61 \pm 0.15$ & 1.61 & 1.61 \\
$0^{++*}$ & 2.8 & 2.38 & 2.64 \\
$0^{++* *}$ & - & 3.11 & 3.64 \\
$0^{++* * *}$ & - & 3.82 & 4.64 \\
$0^{++* * * *}$ & - & 4.52 & 5.63 \\
$0^{++* * * * *}$ & - & 5.21 & 6.62 \\
\hline
\end{tabular}

TABLE I: Four dimensional glueball masses in $\mathrm{GeV}$ with Dirichlet boundary conditions. The value 1.61 of the third and fourth columns is an input taken from lattice results.

\begin{tabular}{|l|c|c|c|c|}
\hline $3 \mathrm{~d}$ State & lattice, $N=3$ & lattice, $N \rightarrow \infty$ & AdS-BH & AdS slice \\
\hline $0^{++}$ & $4.329 \pm 0.041$ & $4.065 \pm 0.055$ & 4.07 & 4.07 \\
$0^{++*}$ & $6.52 \pm 0.09$ & $6.18 \pm 0.13$ & 7.02 & 7.00 \\
$0^{++* *}$ & $8.23 \pm 0.17$ & $7.99 \pm 0.22$ & 9.92 & 9.88 \\
$0^{++* * *}$ & - & - & 12.80 & 12.74 \\
$0^{++* * * *}$ & - & - & 15.67 & 15.60 \\
$0^{++* * * *}$ & - & - & 18.54 & 18.45 \\
\hline
\end{tabular}

TABLE II: Three dimensional glueball masses in units of string tension with Dirichlet boundary conditions. The value 4.07 is an input from lattice. 


\begin{tabular}{|c|c|c|c|}
\hline $\begin{array}{c}\text { Dirichlet } \\
\text { glueballs }\end{array}$ & $\begin{array}{c}\text { lightest } \\
\text { state }\end{array}$ & $\begin{array}{c}1^{\text {st }} \text { excited } \\
\text { state }\end{array}$ & $\begin{array}{c}2^{\text {nd }} \text { excited } \\
\text { state }\end{array}$ \\
\hline $0^{++}$ & 1.63 & 2.67 & 3.69 \\
$2^{++}$ & 2.41 & 3.51 & 4.56 \\
$4^{++}$ & 3.15 & 4.31 & 5.40 \\
$6^{++}$ & 3.88 & 5.85 & 6.21 \\
$8^{++}$ & 4.59 & 5.85 & 7.00 \\
$10^{++}$ & 5.30 & 6.60 & 7.77 \\
\hline
\end{tabular}

TABLE III: Higher spin glueball masses in GeV with Dirichlet boundary condition. The value 1.63 is an input from lattice.

A similar approach was used also for glueball masses in $\mathrm{QCD}_{3}$, taken as dual to scalar fields is $\mathrm{AdS}_{4}$. In this case the Bessel functions are $J_{3 / 2}$ and the mass ratios take the form

$$
\frac{\mu_{p}}{\mu_{1}}=\frac{\chi_{3 / 2, p}}{\chi_{3 / 2,1}} .
$$

Our results for $\mathrm{QCD}_{3}$ are shown in Table II, again compared with lattice [33, 34] and AdS-Schwarzshild [15] results.

For some other results concerning glueball masses using gauge/string duality see for instance [35-38].

\section{HIGHER SPIN STATES AND REGGE TRAJECTORIES}

Recently, very interesting results for the hadronic spectrum were obtained by Teramond and Brodsky[39] considering scalar, vector and fermionic fields in a sliced $A d S_{5} \times S^{5}$ space. It was proposed that massive bulk states corresponding to fluctuations about the $A d S_{5}$ metric are dual to QCD states with angular momenta (spin) on the four dimensional boundary. This way the spectrum of light baryons and mesons has been reproduced from a holographic dual to QCD inspired in the AdS/CFT correspondence.

We used a similar approach to estimate masses of glueball states with different spins[40]. The motivation was to compare the glueball Regge trajectories with the pomeron trajectories. For soft pomerons [41] experimental results show that

$$
J \approx 1.08+0.25 \mathrm{M}^{2} \quad(\mathrm{GeV}) .
$$

It is conjectured that the soft pomerons may be related to glueballs. Recent lattice results are consistent with this interpretation[42].

We assume that massive scalars in the AdS slice with mass $\mu$ are dual to boundary gauge theory states with spin $J$ related by:

$$
(\mu R)^{2}=J(J+4) .
$$

We consider both Dirichlet and Neumann boundary conditions and the results for the four dimensional glueball masses with even spin are shown in tables III and IV respectively.

We found non linear relations between spin and mass squared. We considered linear approximations representing Regge trajectories

$$
J=\alpha_{0}+\alpha^{\prime} M^{2} .
$$

\begin{tabular}{|c|c|c|c|}
\hline $\begin{array}{c}\text { Neumann } \\
\text { glueballs }\end{array}$ & $\begin{array}{c}\text { lightest } \\
\text { state }\end{array}$ & $\begin{array}{c}1^{\text {st }} \text { excited } \\
\text { state }\end{array}$ & $\begin{array}{c}2^{\text {nd }} \text { excited } \\
\text { state }\end{array}$ \\
\hline $0^{++}$ & 1.63 & 2.98 & 4.33 \\
$2^{++}$ & 2.54 & 4.06 & 5.47 \\
$4^{++}$ & 3.45 & 5.09 & 6.56 \\
$6^{++}$ & 4.34 & 6.09 & 7.62 \\
$8^{++}$ & 5.23 & 7.08 & 8.66 \\
$10^{++}$ & 6.12 & 8.05 & 9.68 \\
\hline
\end{tabular}

TABLE IV: Higher spin glueball masses in GeV with Neumann boundary condition. The value 1.63 is an input from lattice.

For Neumann boundary conditions for the states $J^{++}$with $J=$ $2,4, \ldots, 10$ we found

$$
\alpha^{\prime}=(0.26 \pm 0.02) \mathrm{GeV}^{-2} \quad ; \quad \alpha_{0}=0.80 \pm 0.40,
$$

as shown in Figure 1.

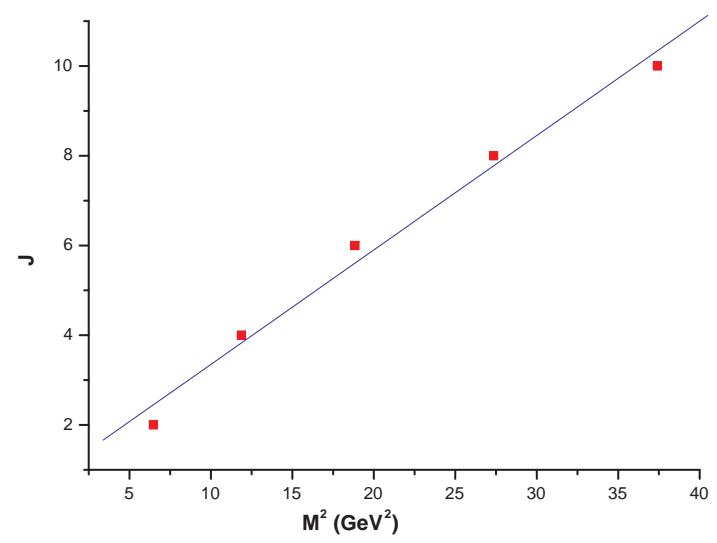

FIG. 1: Spin versus mass squared for the lightest glueball states with Neumann boundary conditions from table IV. The line corresponds to the linear fit.

For Dirichlet boundary conditions, taking the states $J^{++}$with $J=2,4, \ldots, 10$ we found a linear fit with

$$
\alpha^{\prime}=(0.36 \pm 0.02) \mathrm{GeV}^{-2} \quad ; \quad \alpha_{0}=0.32 \pm 0.36,
$$

as shown in Figure 2.

So, Neumann boundary conditions give a glueball trajectory consistent with that of pomerons, eq. (12). These kind of boundary conditions appear in the Randall Sundrum model[43] as a consequence of the orbifold condition.

\section{WILSON LOOPS AND QUARK ANTI-QUARK POTENTIAL}

Confinement criteria for QCD can be discussed with the help of Wilson loops which give the binding energy of the system. Phenomenological results imply that the quark antiquark potential has the form 


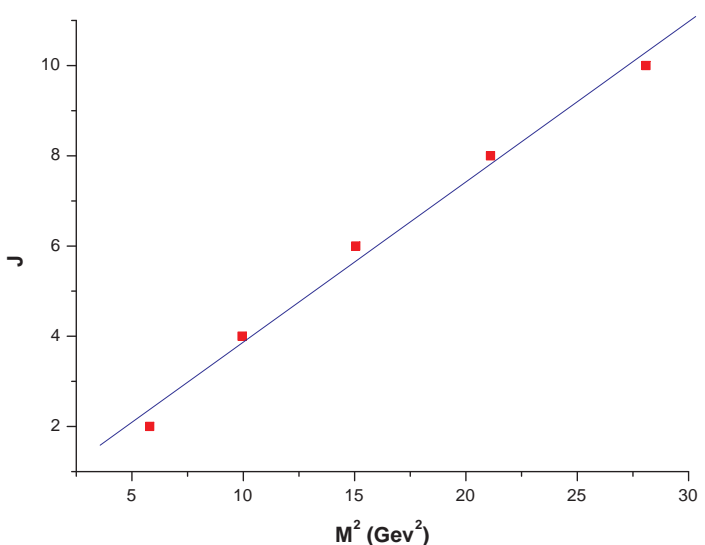

FIG. 2: Spin versus mass squared for the lightest glueball states with Dirichlet boundary conditions from table III. The line corresponds to the linear fit.

$$
E_{\text {Cornell }}(L)=-\frac{4}{3} \frac{a}{L}+\sigma L+\text { constant }
$$

where, $a=0.39$ and $\sigma=0.182 \mathrm{GeV}^{2}$.

In the AdS/CFT correspondence Wilson loops for a heavy quark anti-quark pair in the conformal gauge theory can be calculated from a static string in the AdS space[44, 45]. The corresponding energy is a non confining Coulomb potential.
For an excellent review and extension to other metrics see [46].

We calculated Wilson loops for a quark anti-quark pair in D3-brane space finding different confining behaviors depending on the quark position[47].

Recently we calculated[48] the energy of a static string in an AdS slice between two D3-branes with orbifold condition. The energy for configurations with endpoints on a brane grows linearly for large separation between these points. The derivative of the energy has a discontinuity at some critical separation. Choosing a particular position for one of the branes we find configurations with smooth energy. In the limit where the other brane goes to infinity the energy has a Coulombian behaviour for short separations and can be identified with the Cornell potential eq. (17). This identification leads to effective values for the string tension, the position of the infrared brane and the AdS radius

$$
R=\sqrt{\frac{a}{3 \sigma C_{1}^{2}}}=1.4 \mathrm{GeV}^{-1}
$$

where $C_{1}=\sqrt{2} \pi^{3 / 2} /[\Gamma(1 / 4)]^{2}$.

These results suggest an approximate duality between static strings in an AdS slice and a heavy quark anti-quark configuration in a confining gauge theory.

For other interesting results concerning gauge/string duality and QCD see for instance [49-59].

\section{Acknowledgments}

The authors are partially supported by $\mathrm{CNPq}$ and Faperj.
[1] M. B. Green, J. H. Schwarz, and E. Witten, "Superstring Theory. Vol. 1: Introduction,", Cambridge, 1987.

[2] J. Polchinski, "String theory. Vol. 1: An introduction to the bosonic string," Cambridge 1998.

[3] V. A. Matveev, R. M. Muradian, and A. N. Tavkhelidze, Lett. Nuovo Cim. 7, 719 (1973).

[4] S. J. Brodsky and G. R. Farrar, Phys. Rev. Lett 31 (1973) 1153; Phys. Rev. D 11, 1309 (1975).

[5] J. Polchinski and M. J. Strassler, Phys. Rev. Lett. 88, 031601 (2002).

[6] G. 't Hooft, Nucl. Phys. B 72, 461 (1974).

[7] J. Maldacena, Adv. Theor. Math. Phys. 2, 231 (1998)

[8] S. S. Gubser, I.R. Klebanov, and A.M. Polyakov, Phys. Lett. B 428, 105 (1998).

[9] E. Witten, Adv. Theor. Math. Phys. 2, 253 (1998).

[10] J. M. Maldacena and C. Nunez, Phys. Rev. Lett. 86, 588 (2001).

[11] I. R. Klebanov and A. A. Tseytlin, Nucl. Phys. B 578, 123 (2000).

[12] I. R. Klebanov and M. J. Strassler, JHEP 0008, 052 (2000).

[13] J. Polchinski and M. J. Strassler, "The string dual of a confining four-dimensional gauge theory," arXiv:hep-th/0003136.

[14] E. Witten, Adv. Theor. Math. Phys. 2, 505 (1998).

[15] C. Csaki, H. Ooguri, Y. Oz, and J. Terning, JHEP 9901, 017 (1999).

[16] R. de Mello Koch, A. Jevicki, M. Mihailescu, and J. P. Nunes,
Phys. Rev. D 58, 105009 (1998).

[17] A. Hashimoto, Y. Oz, Nucl. Phys. B 548, 167 (1999).

[18] C. Csaki , Y. Oz, J. Russo, J. Terning, Phys. Rev. D 59, 065012 (1999).

[19] J. A. Minahan, JHEP 9901, 020 (1999).

[20] C. Csaki, J. Terning, AIP Conf. Proc. 494, 321 (1999).

[21] R. C. Brower, S. D. Mathur, C. I. Tan, Nucl. Phys. B 587, 249 (2000).

[22] H. Boschi-Filho and N. R. F. Braga, Phys. Lett. B 560, 232 (2003).

[23] H. Boschi-Filho and N. R. F. Braga, Phys. Lett. B 525, 164 (2002).

[24] S. B. Giddings, Phys. Rev. D 67 , 126001 (2003).

[25] R. C. Brower, C. I. Tan, Nucl. Phys. B 662, 393 (2003).

[26] O. Andreev, Phys. Rev. D 67, 046001 (2003).

[27] J. Polchinski and M. J. Strassler, JHEP 0305 , 012 (2003).

[28] S. J. Brodsky and G. F. de Teramond, Phys. Lett. B 582, 211 (2004).

[29] O. Andreev, Phys. Rev. D 70, 027901 (2004).

[30] O. Andreev, Phys. Rev. D 71, 066006 (2005).

[31] H. Boschi-Filho and N. R. F. Braga, Eur. Phys. J. C 32, 529 (2004).

[32] H. Boschi-Filho and N. R. F. Braga, JHEP 0305, 009 (2003).

[33] C. J. Morningstar and M. Peardon, Phys. Rev. D 56, 4043 (1997). 
[34] M.J. Teper, "Physics from lattice: Glueballs in QCD; topology; SU(N) for all N", arXiv:hep-lat/9711011.

[35] E. Caceres and R. Hernandez, Phys. Lett. B 504, 64 (2001).

[36] R. Apreda, D. E. Crooks, N. Evans, and M. Petrini, JHEP 0405, 065 (2004).

[37] X. Amador and E. Caceres, JHEP 0411, 022 (2004).

[38] E. Caceres and C. Nunez, JHEP 0509, 027 (2005).

[39] G. F. de Teramond and S. J. Brodsky, Phys. Rev. Lett. 94, 201601 (2005).

[40] H. Boschi-Filho, N. R. F. Braga, and H. L. Carrion, Phys. Rev. D 73, 047901 (2006).

[41] P. V. Landshoff, "Pomerons,", published in "Elastic and Difractive Scattering" Proceedings, Ed. V. Kundrat and P. Zavada, 2002, arXiv:hep-ph/0108156.

[42] H. B. Meyer and M. J. Teper, Phys. Lett. B 605, 344 (2005).

[43] L. Randall and R. Sundrum, Phys. Rev. Lett. 83, 3370 (1999); ibid. 83, 4690 (1999).

[44] S. J. Rey and J. T. Yee, Eur. Phys. J. C 22, 379 (2001).

[45] J. Maldacena, Phys. Rev. Lett. 80, 4859 (1998).

[46] Y. Kinar, E. Schreiber, and J. Sonnenschein, Nucl. Phys. B 566, 103 (2000).
[47] H. Boschi-Filho and N. R. F. Braga, JHEP 0503, 051 (2005).

[48] H. Boschi-Filho, N. R. F. Braga, and C. N. Ferreira, Phys. Rev. D 73, 106006 (2006) [Erratum-ibid. D 74, 089903 (2006)].

[49] R. A. Janik and R. Peschanski, Nucl. Phys. B 565, 193 (2000).

[50] R. A. Janik and R. Peschanski, Nucl. Phys. B 586, 163 (2000).

[51] R. A. Janik and R. Peschanski, Nucl. Phys. B 625, 279 (2002).

[52] L. A. Pando Zayas, J. Sonnenschein, and D. Vaman, Nucl. Phys. B 682, 3 (2004).

[53] O. Andreev and W. Siegel, Phys. Rev. D 71, 086001 (2005).

[54] F. Bigazzi, A. L. Cotrone, L. Martucci, and L. A. Pando Zayas, Phys. Rev. D 71, 066002 (2005).

[55] J. Erlich, E. Katz, D. T. Son, and M. A. Stephanov, Phys. Rev. Lett. 95, 261602 (2005).

[56] L. Da Rold and A. Pomarol, Nucl. Phys. B 721, 79 (2005).

[57] N. Evans, J. P. Shock, and T. Waterson, Phys. Lett. B 622, 165 (2005).

[58] S. J. Brodsky and G. F. de Teramond, AIP Conf. Proc. 814, 108 (2006).

[59] S. J. Brodsky and G. F. de Teramond, Phys. Rev. Lett. 96, 201601 (2006). 